\title{
Lusioersily
}

\section{A method for the ECG inverse problem in the frequency domain}

Navarro-Paredes, C., Turner, C., Escalona, OJ., Owens, C., Anderson, JMCC., \& Adgey, J. (2005). A method for the ECG inverse problem in the frequency domain. In Unknown Host Publication (pp. 309-314). (COMPUTERBASED MEDICAL SYSTEMS : PROCEEDINGS OF THE ANNUAL IEEE SYMPOSIUM). IEEE. https://doi.org/10.1109/CBMS.2005.12

Link to publication record in Ulster University Research Portal

\section{Published in:}

Unknown Host Publication

Publication Status:

Published (in print/issue): 15/07/2005

DOI:

10.1109/CBMS.2005.12

\section{Document Version}

Publisher's PDF, also known as Version of record

\section{General rights}

Copyright for the publications made accessible via Ulster University's Research Portal is retained by the author(s) and / or other copyright owners and it is a condition of accessing these publications that users recognise and abide by the legal requirements associated with these rights.

\section{Take down policy}

The Research Portal is Ulster University's institutional repository that provides access to Ulster's research outputs. Every effort has been made to ensure that content in the Research Portal does not infringe any person's rights, or applicable UK laws. If you discover content in the Research Portal that you believe breaches copyright or violates any law, please contact pure-support@ulster.ac.uk. 


\title{
A Method for the ECG Inverse Problem in the Frequency Domain
}

\author{
César Navarro, Colin Turner ${ }^{1}$, Omar Escalona ${ }^{2}$, Colum Owens, \\ John Anderson ${ }^{1}$, A A Jennifer Adgey \\ Regional Medical Cardiology Centre, Royal Victoria Hospital, \\ ${ }^{1}$ University of Ulster, Jordanstown, Northern Ireland \\ ${ }^{2}$ Universidad Simón Bolívar GBBA, Caracas, Venezuela \\ c.navarro@ulster.ac.uk
}

\begin{abstract}
The Inverse ECG problem is ill-conditioned and its solution requires a relatively high computing effort. Additional constraints are required in order to obtain a stable solution. A method is proposed in which the solution of the inverse ECG problem is approached in the frequency domain, taking advantage of the assumption that propagation delays may be ignored and the quasi-periodicity of ECG. In this method usual Tikhonov zero-order constraints are applied to the amplitudes of the signals for a selected frequency domain. This method ensures faster solutions that are spatially and temporally well behaved. Calculation of epicardial electrograms is compared to a basic method.
\end{abstract}

\section{Introduction}

At the Royal Victoria Hospital in Belfast (RVHB), inverse ECG solutions based on epicardial potentials (EP) have been used in retrospective studies. Some promising results have been achieved for the early detection of Acute Myocardial Infarction (AMI) [1,2] and in the localisation of early activation that could help in the treatment of arrhythmias and optimal placement of pacing leads [3]. Calculation of EP must be fast in order to implement some of the developed algorithms in a clinical setting. The inverse ECG problem can be approached in the frequency domain taking advantage of the quasi-static assumption, in which propagation delays may be ignored [4], and the quasi-periodicity of the ECG. Then the resulting method, proposed in this paper, only calculates the amplitudes of signals for a selected range of frequencies. This method uses a Tikhonov regularisation term and the selection of a range of frequencies implicitly imposes constraints that also contribute to a regularised solution. Frequency domain constraints for the inverse ECG problem have already been proposed to penalise high frequency components [5]. The drawback for that formulation is that it requires more complex techniques for dealing with multiple constraints [6].

\section{Materials and methods}

\subsection{Data Collection}

The array of electrodes used for the capture of Body Surface Potentials (BSP) comprises a flexible plastic anterior and posterior electrode harness and a portable recording unit. The anterior harness contains 64 electrodes, including 3 proximal bipolar limb leads (Mason-Likar position), 
and a posterior harness with 16 electrodes. This lead configuration enables recording of 77 unipolar ECG signals with respect to the Wilson central terminal. Recordings are taken over $4198 \mathrm{~ms}$, the sampling rate is $1 \mathrm{kHz}$ and the potentials are recorded using a 12-bit resolution to represent a $5 \mathrm{mV}$ range. The procedure for attaching the harnesses to a patient and taking measurements of electrical potentials lasts about 3-4 minutes.

Data were taken from patients recruited consecutively between February 1999 and February 2001 as they presented via the mobile coronary care unit or emergency department at RVHB. Patients were enrolled throughout the 24 hours if they presented with ischaemic type chest pain for less than 12 hours duration regardless of the presenting 12-lead ECG. All patients had a 12-lead ECG and BSP measured at presentation together with serial cardiac enzymes (creatine kinase and creatine kinase-MB). Patients were excluded if they had pain for less than 20 minutes, or had received fibrinolytic therapy, or nitrates or glycoprotein IIb/IIIa inhibitors prior to the initial 12-lead ECG or BSP, could not give informed consent or BSP were measured 15 minutes after the 12-lead ECG. A total of 427 valid BSP measurements corresponding to the same number of different patients were finally included for the study.

Cardiac technicians recorded 12-lead ECGs and BSP at initial presentation. BSP were uploaded and displayed on an IBM compatible computer running PRIME ${ }^{T M}$ analysis software (Meridian Medical Technologies, Belfast, Northern Ireland). All 80 leads were manually checked and those of unacceptable quality were marked and automatically substituted using linear grid interpolation. Maps with more than 6 bad leads were disregarded to avoid inappropriate reliance on interpolated data. Global QRS onset, offset and T wave offset were marked. Baseline wander and $50 \mathrm{~Hz}$ line noise were removed.

\subsection{A basic method for Inverse ECG}

The mathematical formulation for solving the forward and inverse ECG problems using the quasi-static assumption can be found in the literature [7]. Briefly, a transfer matrix $\mathbf{U}$ relates $N$ body surface potentials $\Phi_{B}$ to $M$ epicardial potentials $\Phi_{H}$ by a linear equation given by (1). Given a particular $\Phi_{B}$, the corresponding epicardial potentials $\Phi_{H}$ can be calculated (using a zero order Tikhonov regularisation [7]) according to equation (2), where $\gamma$ is the non-negative regularisation parameter, $\mathbf{I}$ is the $M \times M$ identity matrix and superscripts $T$ and -1 mean transpose and inverse respectively.

$$
\begin{aligned}
& \Phi_{B}=\mathbf{U} \Phi_{H} \\
& \Phi_{H}=\left(\mathbf{U}^{T} \mathbf{U}+\gamma \mathbf{I}\right)^{-1} \mathbf{U}^{T} \Phi_{B}
\end{aligned}
$$

The transfer matrix $\mathbf{U}$ was calculated according to [8] using a general thoracic model which is based on the male cadaver from the visible human project [9]. A 3-D mesh consisting of 11404 tetrahedral elements, 1136 torso surface nodes and 59 epicardial nodes was constructed. Conductivities of lungs, spine, sternum and fat layer were taken from literature [1].

For the initial implementation and validation of a basic method for inverse ECG, calculations were carried out using 20 static epicardial distributions generated by dipole and multipole configurations within the geometry of the heart. These distributions were taken as "real" data in order to make comparisons with the calculated EP. The optimal regularisation parameter was taken from the point of maximum curvature for the L-curve. The algorithm reconstructed EP using the corresponding BSP with and without generated noise. Noise was added so that every measurement could have up to $30 \%$ of the maximum EP according to a uniform distribution of probability. As a result of these previous tests, it was found that inverse calculations had virtually no error in the absence of 
noise in which the regularisation parameter is set to zero. Otherwise many forward problems need to be solved in order to construct an L-curve and hence choose the optimal regularisation parameter [10].

In order to improve the search of the optimal regularisation parameter $\left(\gamma^{*}\right)$ the Minimal Product method (MINP) [11] is preferred instead of the L-curve analysis since it is easier to implement and equally effective. In this method, $\gamma^{*}$ minimises the product of the norm of the solution and the norm of the residual given by equation (3).

$$
P(\gamma)=\left\|\Phi_{H}\right\|\left\|\Phi_{B}-\mathbf{U} \Phi_{H}\right\|
$$

the dependency of $\gamma$ is given by equation (2).

The accuracy of the MINP was checked against the analysis of the L-curve for the 20 test epicardial distributions and the results were in keeping with those reported in the literature [11, 12]. For the search of $\gamma^{*}$, an initial value $\left(\gamma_{0}\right)$ is proposed which is used to define an initial interval $\left[0, \gamma_{0}\right]$ where the optimal value can be found. This initial value is given by:

$$
\gamma_{0}=\omega\left\|\Phi_{B}-\widetilde{\mathbf{U} \Phi_{H}}\right\| /\left\|\Phi_{B}\right\|
$$

where the residual involves $\widetilde{\Phi_{H}}$ which are the epicardial potentials calculated using $\gamma=0$ and $\omega$ is a constant of proportionality. $\left\|\Phi_{B}-\widetilde{\mathbf{U} \Phi_{H}}\right\|=0$ is in keeping with the case of absence of error in which $\gamma^{*}$ is zero. Special attention must be taken in cases in which $\left\|\Phi_{B}\right\|$ is close to zero. The extreme case is when $\left\|\Phi_{B}\right\|=0$ (for instance during the isoelectric line of the ECG) and $\left\|\Phi_{H}\right\|$ is expected to be zero as well. In this case, according to (2) $\gamma$ could be any value.

As $\gamma^{*}$ minimises $P(\gamma), d P\left(\gamma^{*}\right) / d \gamma=0$. Now $d P(0) / d \gamma<0$, and $\omega$ is chosen (using experimental data) so that $d P\left(\gamma_{0}\right) / d \gamma>0$; so the turning point is somewhere in the interval $\left(0, \gamma_{0}\right)$. Then the length of the interval is reduced using successive midpoints to find $\gamma^{*}$.

The basic algorithm for the inverse ECG problem is then:

1. Read the input data $\Phi_{B}$ (the torso surface measurements).

2. Calculate $\widetilde{\Phi_{H}}$ (using $\gamma=0$ in equation (2)).

3. Calculate $\gamma_{0}$ using equation (4).

4. Reduce the interval $\left[0, \gamma_{0}\right]$ in order to find $\gamma^{*}$ according to the proposed steps.

5. Present the calculation of $\Phi_{H}$ using $\gamma^{*}$ as the regularised solution.

One variant of this algorithm is to check if $\left\|\Phi_{B}\right\|$ is too close to zero according to a threshold. In such a case the optimal regularisation parameter is taken as the last calculated $\gamma^{*}$ and steps 2 to 4 are avoided. The threshold was empirically set to $30 \%$ of the maximum $\left\|\Phi_{B}\right\|$.

The calculations of EP by mean of this basic algorithm were carried out using data from 427 patients previously described. Data from 5 randomly chosen patients was used for setting $\omega$ to 0.01 so that $\gamma_{0}$ allows to find $d P\left(\gamma_{0}\right) / d \gamma>0$ in two iterations.

\subsection{The proposed method}

In this section the proposed method in the frequency domain is described. At the end once the problem is converted to the frequency domain this method uses the basic method described in the previous section.

Fluctuations of the potential at the node $i$ on the torso surface $\Phi_{B i}(t)$ can be expressed in terms of a Fourier series or in practical terms it can be expressed as a summation as:

$$
\Phi_{B i}(t)=a_{B i 0}+\sum_{k=1}^{K}\left(a_{B i k} \cos \left(2 \pi k f_{0} t\right)+b_{B i k} \sin \left(2 \pi k f_{0} t\right)\right) \quad i=1 \ldots N
$$


where $f_{0}$ is the fundamental frequency and $K$ is the number of terms. Eq. (5) can be rewritten as:

$$
\Phi_{B i}=\mathbf{a}_{B i} \mathbf{c}^{T}+\mathbf{b}_{B i} \mathbf{s}^{T} \quad i=1 \ldots N
$$

in which the notation for time dependancy of $\Phi_{B i}$ is omitted for simplicity and:

$\mathbf{a}_{B i}=\left[a_{B i 0}, a_{B i 1}, \ldots, a_{B i K}\right], \quad \mathbf{c}=\left[1, \cos \left(2 \pi f_{0} t\right), \cos \left(2 \pi 2 f_{0} t\right), \ldots, \cos \left(2 \pi K f_{0} t\right)\right]$,

$\mathbf{b}_{B i}=\left[b_{B i 1}, b_{B i 2}, \ldots, b_{B i K}\right] \quad$ and $\quad \mathbf{s}=\left[\sin \left(2 \pi f_{0} t\right), \sin \left(2 \pi 2 f_{0} t\right), \ldots, \sin \left(2 \pi K f_{0} t\right)\right]$.

Similarly, the potential at the node $j$ on the epicardium $\Phi_{H j}(t)$ can be expressed according to:

$$
\Phi_{H j}=\mathbf{a}_{H j} \mathbf{c}^{T}+\mathbf{b}_{H j} \mathbf{s}^{T} \quad j=1 . . M
$$

where $\mathbf{a}_{H j}=\left[a_{H j 0}, a_{H j 1}, \ldots, a_{H j K}\right] \quad$ and $\quad \mathbf{b}_{H j}=\left[b_{H j 1}, b_{H j 2}, \ldots, b_{H j K}\right]$.

Then, according to equation (1) the torso surface potential for the node $i$ is given by:

$$
\Phi_{B i}=\mathbf{u}_{i} \Phi_{H}
$$

where $\mathbf{u}_{i}$ is the $i$ th row of the transfer matrix $\mathbf{U}$. Equation (8) can be rewritten as

$$
\Phi_{B i}=u_{i 1} \Phi_{H 1}+u_{i 2} \Phi_{H 2}+\ldots+u_{i M} \Phi_{H M}
$$

using (7) and (9):

$$
\Phi_{B i}=u_{i 1}\left(\mathbf{a}_{H 1} \mathbf{c}^{T}+\mathbf{b}_{H 1} \mathbf{s}^{T}\right)+\ldots+u_{i M}\left(\mathbf{a}_{H M} \mathbf{c}^{T}+\mathbf{b}_{H M} \mathbf{s}^{T}\right)
$$

rearranging terms in (10), $\Phi_{B i}$ can be expressed as:

$$
\Phi_{B i}=\left[\mathbf{u}_{i} \alpha_{0}, \mathbf{u}_{i} \alpha_{1}, \ldots, \mathbf{u}_{i} \alpha_{K}\right] \mathbf{c}^{T}+\left[\mathbf{u}_{i} \beta_{1}, \mathbf{u}_{i} \beta_{2}, \ldots, \mathbf{u}_{i} \beta_{K}\right] \mathbf{s}^{T}
$$

where $\alpha_{k}=\left[a_{H 1 k}, a_{H 2 k}, \ldots, a_{H M k}\right]^{T} \quad k=0 . . K$ and $\beta_{k}=\left[b_{H 1 k}, b_{H 2 k}, \ldots, b_{H M k}\right]^{T}$ $k=1 . . K . \alpha_{k}$ and $\beta_{k}$ are vectors whose components are the $M$ cosine and sine coefficients respectively corresponding to the frequency $k f_{0}$. Then equating (6) and (11) and assuming $\beta_{0}=\mathbf{0}$ it can be obtained:

$$
a_{B i k}=\mathbf{u}_{i} \alpha_{k} \quad b_{B i k}=\mathbf{u}_{i} \beta_{k} \quad i=1 . . N \quad k=0 . . K
$$

According to equations (12), for a particular frequency $k f_{0}$ the torso surface cosine and sine coefficients are respectively related to the epicardial cosine and sine coefficients by means of the transfer matrix. Grouping the cosine and sine coefficients of the $N$ torso surface nodes for a particular frequency $k f_{0}$, two vectors $\mathbf{v}_{k}$ and $\mathbf{w}_{k}$ can be formed:

$$
\mathbf{v}_{k}=\left[a_{B 1 k}, a_{B 2 k}, \ldots, a_{H N k}\right]^{T}, \quad \mathbf{w}_{k}=\left[b_{B 1 k}, b_{B 2 k}, \ldots, b_{B N k}\right]^{T}
$$

then equation (12) can be written as:

$$
\mathbf{v}_{k}=\mathbf{U} \alpha_{k} \quad, \mathbf{w}_{k}=\mathbf{U} \beta_{k} \quad k=0 . . K
$$

which is similar to equation (1) and for solving the inverse ECG problem. Equation (2) is used replacing the corresponding frequency vectors for the potentials vectors and the basic method described in section (2.2) was applied.

The calculations of EP using the proposed method were carried out using data from 427 patients previously described. Again data from 5 randomly chosen patients was used for setting $\omega$ to 0.1 so that $\gamma_{0}$ allows to find $d P\left(\gamma_{0}\right) / d Y>0$ in two iterations. Fast Fourier Transform (FFT) and Inverse FFT were used for switching from time to frequency domain and viceversa. The number of samples for each of the 80-lead electrodes was reduced from 4198 to 4096. 


\section{Results}

Table 1. Summarised results for inverse ECG calculations using the proposed and a basic method

\begin{tabular}{rrrrrr}
\hline & \multicolumn{2}{c}{ Fourier based method } & \multicolumn{3}{c}{ Basic method } \\
& forward problems & time $(\mathrm{s})$ & forward problems & time $(\mathrm{s})$ & $d(\%)$ \\
\hline Average & 1983 & 20.3 & 15164 & 108.1 & 4.94 \\
Tipical dev & 1156 & 10.9 & 19840 & 135.7 & 2.04 \\
Max & 9876 & 114.0 & 304165 & 2054.0 & 14.38 \\
Min & 486 & 8.0 & 4010 & 30.0 & 1.33 \\
\hline
\end{tabular}

If the optimal regularisation parameter $\gamma^{*}$ is known, $2 K$ calculations would be required using the proposed method in comparison to 4096 calculations using the basic method. Final calculation of $\Phi_{H}$ requires previous calculations involving equation (2) and solving forward problems using equation (1). Particularly for the study presented in this paper these previous calculations allow the calculation of the product given by equation (3) which leads to $\gamma^{*}$. Assuming the same amount of previous calculations for obtaining $\gamma^{*}$, the ratio $\frac{2 K}{4096}$ is given by $\frac{2 f}{r}$ where $f$ is the maximum frequency and $r$ is the sampling rate of $1000 \mathrm{~Hz}$. Then for example, if frequencies are taken up to $100 \mathrm{~Hz}$, the proposed method theoretically is 5 times quicker. Methods presented in sections (2.2) and (2.3) were tested using data from 427 patients described in section (2.1) and for frequencies up to $100 \mathrm{~Hz}$. The difference $d$ is calculated according to equation (15) for the comparison of epicardial electrograms and results are summarised in table (1). Algorithms have been implemented in a PC using $\mathrm{C}$ language. Times are in seconds and the label "forward problems" denotes the number of previous forward problems required to find $\gamma^{*}$. Figures have been rounded when required for easier reading and comparison. Time for converting from time to frequency domain and viceversa was around $2 \mathrm{~s}$. Average difference is less than 5\% and the proposed method requires $20.3 \mathrm{~s}$ in average for calculating 59 epicardial electrograms using $77 \mathrm{BSP}$ in comparison to $108.1 \mathrm{~s}$ required by a basic method for the same task. Then it is about 5 times faster and this result is in keeping to the simplified theoretical situation.

$$
d=\left(\frac{1}{59} \sum_{j=1}^{59}\left(\max \left(\Phi_{H j}^{*}(t)\right)-\min \left(\Phi_{H j}^{*}(t)\right)^{-1} \sum_{t=1}^{4096}\left|\Phi_{H j}^{*}(t)-\Phi_{H j}(t)\right|\right) 100 \%\right.
$$

where $\Phi_{H j}^{*}(t)$ and $\Phi_{H j}(t)$ are the vectors of epicardial potentials calculated using the basic and proposed methods from sections (2.2) and (2.3) respectively.

\section{Discussion}

The proposed method for solving the Inverse ECG problem in the frequency domain is consistently faster than a basic method and the average difference is less than $5 \%$ when normalised electrograms are compared. These faster calculations are required in an acute setting where decision making has to be fast. Particularly for the early detection of AMI a rapid and reliable diagnosis improve the prognosis and treatment of the patients.

The average difference of $4.94 \%$ shown in table (1) needs to be addressed in terms of the effectiveness of the calculated EP for interpretation and diagnosis according to previously developed algorithms [1, 2]. For instance it will be important to asses how the calculated EP using the proposed method behaves for the ST-T segment (of relative low magnitude in comparison to QRS) which is important for AMI detection. Another important test will be the evaluation of calculated 
activation times which are useful in the construction of isochrone maps with a potential application in a catheterisation laboratory [3].

Another limitation of this study is that the accuracy of calculations of EP have been only validated using simulated data generated from dipoles and multipole configurations within a model of the heart. Data from animal preparations in which EP could be measured simultaneously when BSP are taken, would guide in finding not only a fast but also an accurate method. So far EP calculations present an explanation of the cardiac sources in terms of the governing physical laws of the BSP measurements and they offer promising ways of improving on interpretation of 12-lead ECG and BSP for various clinical applications.

\section{Conclusion}

In practical terms, a significant saving in calculation time may be obtained as a result of attempting the solution of the Inverse ECG problem in the frequency domain. Then these faster solutions are in keeping with a clinical setting where applications based on EP are encouraging.

\section{References}

[1] C. Navarro, C. Owens, J. Riddell, A. McClelland, J. M. Anderson, O. Escalona, C. Turner, and J. Adgey. The use of calculated epicardial potentials improves significantly the sensitivity of a diagnostic algorithm in the detection of acute myocardial infarction. Journal of Electrocardiology, 36 (S1):127-132, December 2003.

[2] C. Owens, C. Navarro, A. McClelland, J. Riddell, O. Escalona, J. M. Anderson, and J. Adgey. Improved detection of acute myocardial infarction using a diagnostic algorithm based on calculated epicardial potentials. Submitted to American Heart Journal, 2005.

[3] A. McClelland. MD thesis to be submitted to the Faculty of Medicine and Health Sciences Queens University. Belfast, Northern Ireland, 2005.

[4] R. Plonsey. Laws governing current flow in the volume conductor. In C. V. Nelson and D. B. Geselowitz, editors, The theoretical basis of electrocardiology, pages 165-174. Clarendon Press, Oxford, 1976.

[5] K. G. Srinidhi, D. H. Brooks, and R. S. MacLeod. Imposition of Frequency Domain Constraints in Inverse Electrocardiography. In The First Joint BMES-EMBS Conference Serving Humanity, Advancing Technology, page 277 , Atlanta, GA USA, 1999.

[6] D. H. Brooks, G. F. Ahmad, and R. S. MacLeod. Inverse Electrocardiography by Simultaneous Imposition of Multiple Constraints. IEEE Transactions on Biomedical Engineering, 46(1):3-18, January 1999.

[7] R. M. Gulrajani. The Forward and Inverse Problems of Electrocardiography. IEEE Engineering in Medicine and Biology, pages 84-101, September/October 1998.

[8] Y. Yamashita and T. Takahashi. Use of the Finite Element Method to Determine Epicardial from Body Surface Potentials Under a Realistic Torso Model. IEEE Transactions on Biomedical Engineering, BME-31(9):611-621, September 1984.

[9] M. J. Ackerman. The visible human project. In Proceedings of the IEEE, volume 86, pages 504-511, March 1998.

[10] C. Navarro-Paredes. Calculated Epicardial Potentials for Early Diagnosis of Acute Myocardial Infarction. PhD thesis, School of Mechanical and Electrical Engineering, University of Ulster, Jordanstown, Newtownabbey. Co. Antrim, Northern Ireland, 2003.

[11] J. Lian, D. Yao, and B. He. A New Method for Implementation of Regularization in Cortical Potential Imaging. In Proceedings of the 20th Annual Int. Conf. IEEE/EMBS, volume 20, pages 2155-2158, Hong Kong, 1998.

[12] P. R. Johnston and R. Gulrajani. Selecting the corner in the L-curve approach to Tikhonov regularization. IEEE Transactions on Biomedical Engineering, 47(9):1293-1296, September 2000. 